\title{
MONTE CARLO MODELLING OF THE EFFECTIVE DIFFUSIVITY IN COMPOSITE MATERIAL
}

\author{
I.V. Belova ${ }^{1}$ and G.E. Murch ${ }^{1}$ \\ ${ }^{1}$ Diffusion in Solids Group \\ School of Engineering \\ The University of Newcastle, Callaghan \\ New South Wales, Australia 2308 \\ a Irina.Belova@newcastle.edu.au, ${ }^{b}$ Graeme.Murch@newcastle.edu.au
}

Keywords: Diffusion, Monte Carlo, Composites, Effective diffusivity,

\begin{abstract}
In this paper we perform a systematic Monte Carlo study of the effective diffusivity in a 2D composite in which the dispersed phase (squares) is arranged in square planar and brick-work patterns within the matrix phase. We focus on the commonly encountered case where the dispersed phase has a much lower diffusivity than the matrix and where the segregation of the diffusant favours the matrix phase. It is found that while the generalized Maxwell-Garnett Equation generally describes the effective diffusivity semi-quantitatively, in order to have an accurate representation of the effective diffusivity at moderate volume fractions of the dispersed phase, it is necessary to use a more refined Maxwell-Garnett Equation that takes into account the actual shape and geometry of the dispersed phase.
\end{abstract}

\section{Introduction}

Models in which spherical, cubic and other shaped particles (having one diffusivity) are dispersed in a matrix (having another diffusivity) have found many applications in science and engineering ranging from diffusion in porous media, to diffusion in two-phase material, to diffusion in nanocrystalline material. The chief problem is to calculate the effective diffusivity of the whole, as a function of the volume fraction of the dispersed phase. Furthermore, there are many analogues of the problem that are also of great interest including the calculation of the effective magnetic permeability, effective thermal diffusivity and effective electrical conductivity all of which have very closely related mathematical behaviour. A rather commonly found situation occurs when 1) the dispersed phase has a diffusivity that is considerably lower than the matrix and 2) the concentration of the diffusant is considerably lower in the dispersed phase compared with the matrix phase. An extreme example of these conditions is found when the dispersed phase is impermeable to the diffusant, indeed, much of the older literature deals with this [1].

Much of the recent literature to calculate the effective diffusivity has been concerned with various extended forms of the original Maxwell [2] and Maxwell-Garnett [3] equations that were derived over a century ago. Making use of a concentric sphere model and phenomenological diffusion arguments, Kalnin et al. [4,5] were able to generalize these equations to take into account the possible segregation of diffusant between the two phases (we will refer to the host or matrix phase as phase 1 and the dispersed phase as phase 2) to obtain for the effective diffusivity $D_{\text {eff }}$ :

$$
D_{e f f}=\frac{s D_{1}\left[(1-\phi+d \phi) D_{2}+s(1-\phi) D_{1}(d-1)\right]}{(\phi+s(1-\phi))\left[s D_{1}(d-1+\phi)+(1-\phi) D_{2}\right]}
$$

where $d$ is the dimension, $D_{1}$ is the diffusivity of a diffusant in the matrix phase and $D_{2}$ is the diffusivity of the same diffusant in the dispersed phase, $\phi$ is the volume fraction of the dispersed phase, and $s$ is the segregation factor, sometimes called the segregation coefficient, of the diffusant 
(the ratio of the equilibrium concentration of the diffusant in the matrix phase $\left(c_{1}\right)$ to that in the dispersed phase $\left(c_{2}\right)$. The principal assumption in deriving Eq. 1 is that it refers to a hypothetical diffusion situation where the two phases are arranged alternately in all directions. It contains no explicit information about the actual arrangement of the particles of the dispersed phase or of their shape. More refined equations that are related to the Maxwell-Garnett equation have also been derived to deal with specific square-planar and brick-work geometries of cubic and square particles of the dispersed phase and these have been in very good agreement with the limited available Monte Carlo data [6]. However, another recent limited Monte Carlo study implied that such refinements are unnecessary since in fact Eq. 1 gives better agreement with the results for the limiting case of the effective diffusivity in a 2D composite with an impermeable dispersed phase (square and circular particles in different arrangements [5]).

In order to determine the usefulness of Eq. 1 and to evaluate the more refined equations the present paper reports on a systematic Monte Carlo study of the effective diffusivity for a 2D composite consisting of square dispersed particles arranged in square-planar and brick-work patterns. We have focussed on the condition when the diffusivity is one thousand times larger in the matrix phase than the dispersed phase and we have then varied the degree of segregation of the diffusant between the matrix phase (favoured) and the dispersed phase.

\section{Models and Theory}

We address the two-dimensional model where squares (the dispersed phase) are arranged in square-planar and a brick-work arrangements as shown in Figs. 1a,b. If we denote the thickness of matrix between the dispersed phase by $\delta$ and the period by $2 L$ then the (area) fraction of such regions (not including those regions parallel to the $x$-direction) is simply given by $\varepsilon_{\mathrm{x}}=\delta / 2 L$ (see Fig. 1a) and analogously for $\varepsilon_{\mathrm{y}}$. Since we have squares, then $\varepsilon_{\mathrm{x}}=\varepsilon_{\mathrm{y}}=\varepsilon$ and the overall volume fraction of inclusions is simply given by:

$$
\phi=(1-\varepsilon)^{2}
$$

For the square planar arrangement (See Fig. 1a), $D_{\text {eff }}$ has been derived from geometrical considerations [6]:

$$
D_{e f f}=\frac{s D_{b}\left(\varepsilon(1-\varepsilon) s D_{b}+(\phi+\varepsilon) D_{l}\right)}{(\phi+s(1-\phi))\left[\varepsilon D_{l}+(1-\varepsilon) s D_{b}\right]}
$$

For the brick-work type arrangement (see Fig. 1b), $D_{\text {eff }}^{x}$ and $D_{\text {eff }}^{y}$ have been derived from geometrical considerations [6]:

$$
\begin{aligned}
& D_{\text {eff }}^{x}=\frac{s D_{b}\left(\varepsilon(1-\varepsilon) s D_{b}+(\phi+\varepsilon)(0.25+\phi+\varepsilon) D_{l}\right)}{(0.25+\phi+\varepsilon)(\phi+s(1-\phi))\left[\varepsilon D_{l}(0.25+\phi+\varepsilon)+(1-\varepsilon) s D_{b}\right]} \\
& D_{e f f}^{y}=\frac{s D_{b}\left(\varepsilon(1-\varepsilon) s D_{b}+(\phi+\varepsilon) D_{l}\right)}{(\phi+s(1-\phi))\left[\varepsilon D_{l}+(1-\varepsilon) s D_{b}\right]}
\end{aligned}
$$


(a)

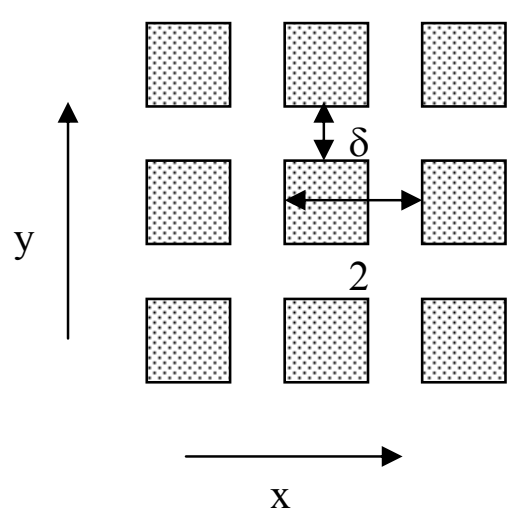

(b)

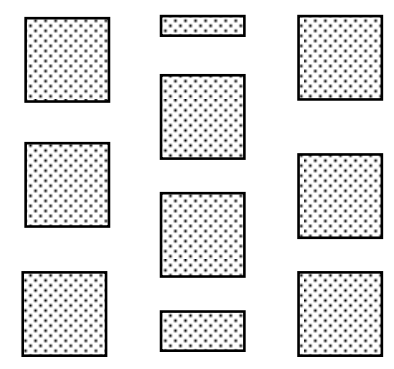

Fig. 1. Schematic representation of two-dimensional composite comprising square low diffusivity dispersed phase particles surrounded by a high diffusivity matrix phase. (a) - square-planar arrangement, (b) - brick-work arrangement.

\section{Computer simulation}

In order to address the problem we represented the diffusivities in the two individual phases by making use of the formal equivalence of the Fick and Einstein definitions of the diffusivity. We mapped a periodic unit cell of the square-planar and brick-work arrangements (see Fig. 1a,b) of squares onto a very fine mesh array $(600 \mathrm{x} 600)$ that was then explored by randomly walking particles released one at a time and directed by standard Monte Carlo diffusion computer simulation methods. The array was in effect a very fine-grained replica of the original spatial arrangement of squares. Testing showed that the arrays used were sufficiently fine-grained to avoid coarse-graining effects. This array has no atomistic meaning of course: it is purely an artifice to enable the macroscopic diffusivity to be conveniently represented by means of a diffusivity based on random walks of test particles on a lattice. The diffusivities were scaled directly to the jump frequencies from site-to-site within each phase, time was scaled to the number of jump attempts of a particle. The locations where the test particles were originally released in the array were weighted according to the designated value of the segregation factor. This is a very important step that must not be overlooked. The diffusivity was determined directly from the Einstein Equation (in 2D):

$$
D=<R^{2}>/ 4 t
$$

where $R$ is the displacement of a particle after a long time $t$ and the Dirac brackets indicate an average over a large number of particle histories, in this case we used $10^{5}$. The number of jump attempts was set at $10^{6}$ : testing showed that this was adequate to obtain long time limit diffusion behaviour.

In principle, instead of using Eq. 6 it would be possible to use the Fickian definition of the diffusivity by injecting the particles one by one at a single line (in $2 \mathrm{D}$ ), or a plane in $3 \mathrm{D}$, and determining the effective diffusivity by processing the concentration profile of particles after some time $t$ according to an appropriate (semi-infinite solid) solution to the Diffusion Equation (Fick's Second Law) or by setting up a steady-state condition by eliminating particles at another line (in 2D), or a plane in 3D, and then calculating the effective diffusivity directly from Fick's First Law. We believe that for this problem the Einstein method is superior because, by fully employing periodic boundaries in all directions, it avoids possible surface effects. 


\section{Results and discussion}

(a)

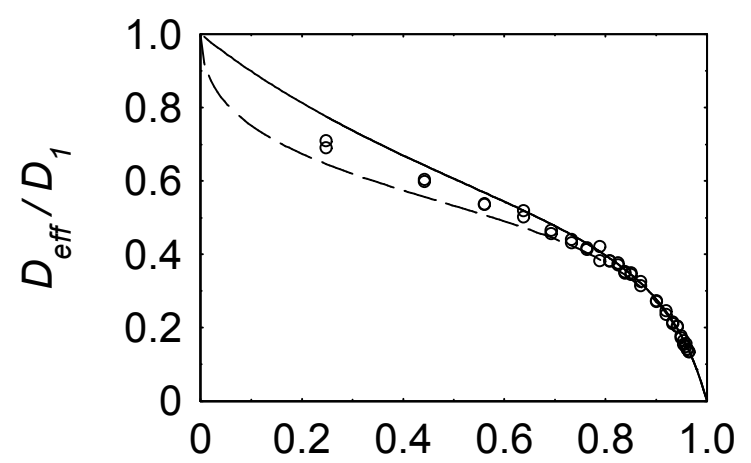

(c)

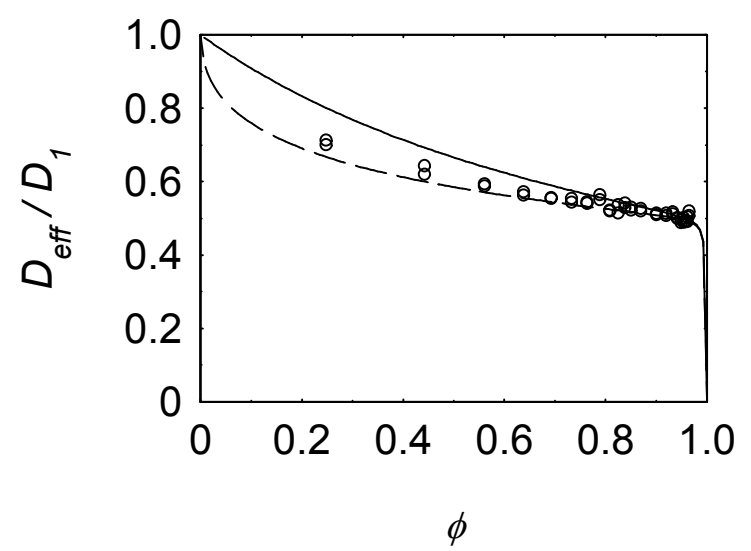

(b)

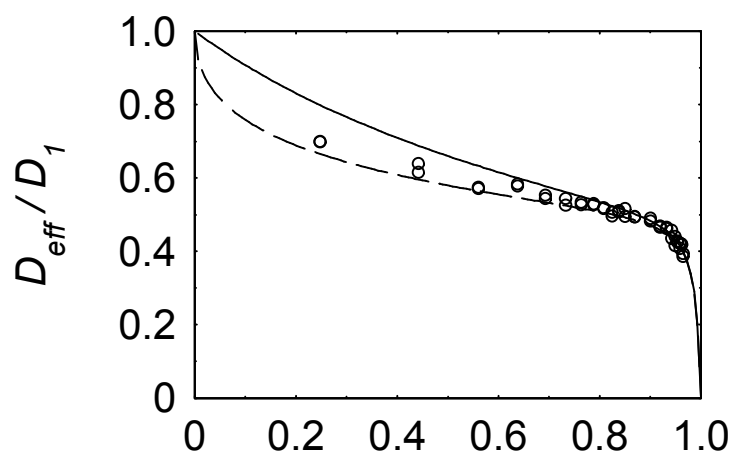

(d)

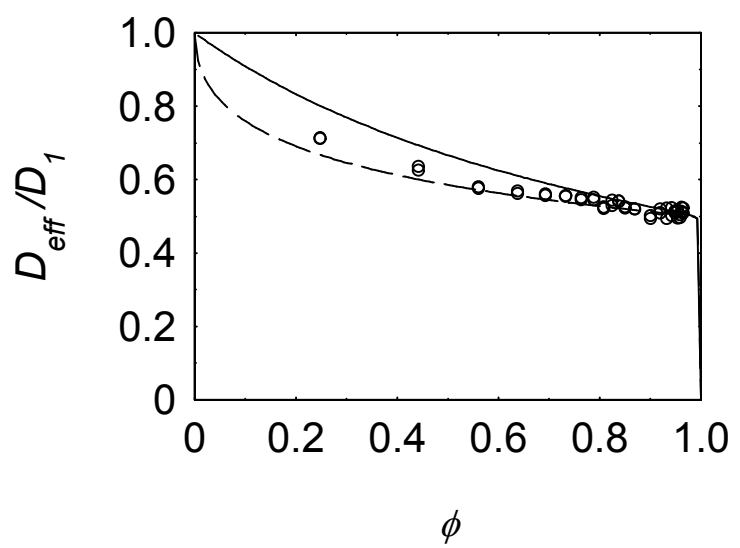

Fig. 2. Ratio of the effective diffusivity to the matrix diffusivity as a function of the area fraction of the dispersed phase $\phi$ for the case of squares in a square-planar arrangement. Data points are Monte Carlo calculations, solid lines: Eq. 1, dashed lines represent the estimate from Eq. 3. (a) segregation factor $\mathrm{s}=10$, (b) $\mathrm{s}=100$; (c) $\mathrm{s}=1000$; (d) $\mathrm{s}=10^{4}$. The ratio of the matrix diffusivity to the dispersed phase diffusivity is $10^{3}$.

In Figs. 2a-d we present result for the effective (scaled) diffusivity in a square-planar arrangement of squares (dispersed phase) as a function of area fraction of the dispersed phase for four values of the segregation factor. It can be seen that both Eqs. 1 and 3 converge at very high fractions of the dispersed phase and also give excellent agreement with the Monte Carlo results. At lower fractions of the dispersed phase it can be clearly seen that Eq. 3 gives superior agreement with the Monte Carlo data. Very low fractions of the dispersed phase were not accessible using the present algorithm but we might anticipate that in that case Eq. 1 would actually give superior agreement. The reason is that including the effect of geometry in the fashion used to derive Eq. 3 would, in all probability, give too exaggerated an effect at low fractions of the dispersed phase. 
(a)

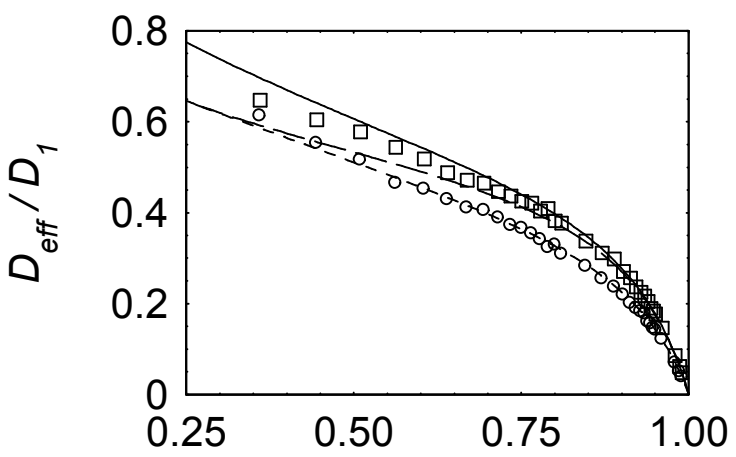

(c)

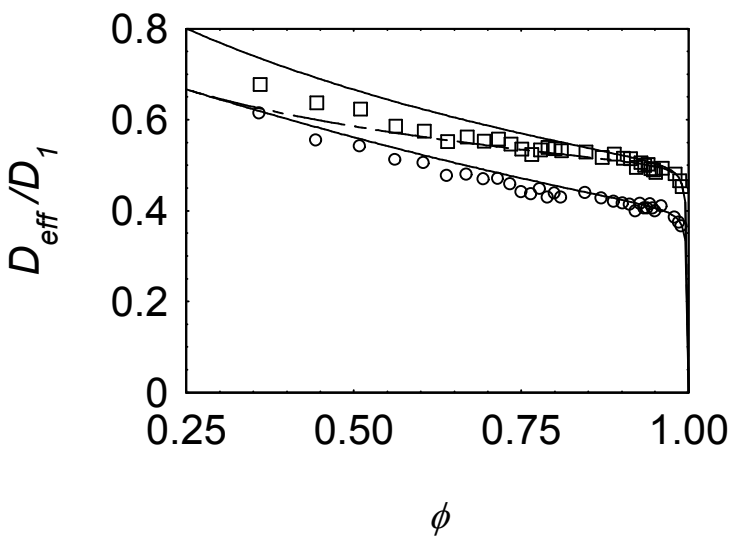

(b)

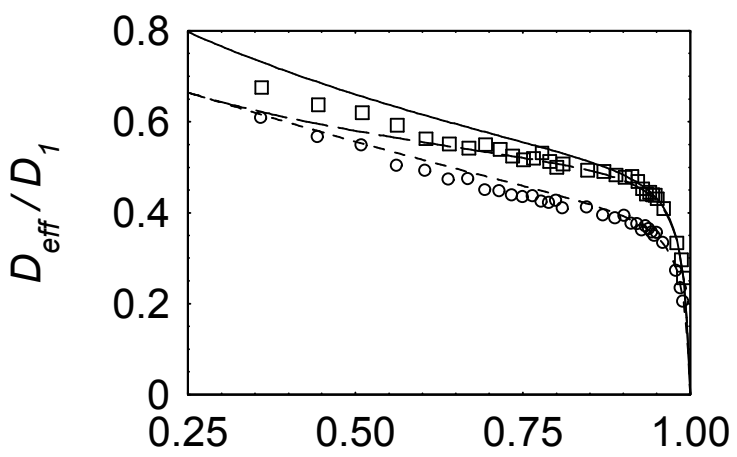

(d)

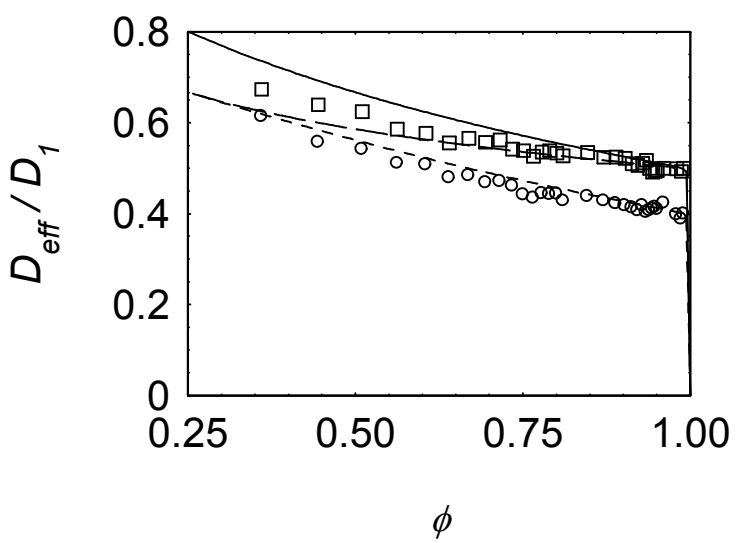

Fig. 3. Ratio of the effective diffusivity to the matrix diffusivity as a function of the area fraction of the dispersed phase $\phi$ for the case of squares in a brick-work type arrangement (see Fig. 1). Data points are Monte Carlo calculations: open circles - diffusivity in the x-direction; open squares diffusivity in the y-direction; solid lines represent the Maxwell estimate (Eq. 1), long-dashed lines represent estimate by Eq. 4 and the short-dashed lines - estimate by Eq. 5. (a) - segregation factor $\mathrm{s}=10$, (b) $-\mathrm{s}=100$; (c) $\mathrm{s}=10^{3}$; (d) $\mathrm{s}=10^{4}$. The ratio of the matrix diffusivity to the dispersed phase diffusivity is $10^{3}$.

In Fig. 3a-d we present results for the effective (scaled) diffusivity in a brick-work arrangement of squares (dispersed phase) as a function of area fraction of the dispersed phase for four values of the segregation factor. In this case, the diffusivity is of course anisotropic. At high area fractions of the dispersed phase it can be seen that Eqs. 1 and 4 converge to the Monte Carlo results for diffusion in the y direction. At lower dispersed phase fractions Eq. 1 is seen to be incapable of describing the effective diffusivity except perhaps as a rough estimate for the effective diffusivity in the $\mathrm{y}$ direction. Eqs. 4 and 5 describe the diffusivity in the $\mathrm{x}$ and $\mathrm{y}$ directions respectively quite well at all area fractions above 0.4. Below this fraction, these two equations start to converge whereas the Monte Carlo data for the two directions still remain separated. Very low fractions of the dispersed phase were not accessible using our algorithm. It would be anticipated however that in this region the actual geometry of the arrangement of squares would become less important, that the effective diffusivity for the $\mathrm{x}$ and $\mathrm{y}$ directions would in fact converge and that Eq. 1 would probably then give the best agreement. 


\section{Conclusions}

We have performed a systematic Monte Carlo study of the effective diffusivity in a 2D composite in which the dispersed phase (squares) was arranged in square-planar and brick-work patterns within the matrix. We focused on the commonly encountered case where the dispersed phase has a much lower diffusivity than the matrix and where the segregation of the diffusant favours the matrix phase. It was found that while the generalized Maxwell-Garnett Equation [4,5] will always describe the effective diffusivity semi-quantitatively, in order to have an accurate representation of the effective diffusivity at moderate volume fractions of the dispersed phase, it is necessary to use a more refined Maxwell-Garnett Equation that takes into account the actual shape and geometry of the dispersed phase.

\section{Acknowledgement}

We wish to thank the Australian Research Council for its support of this research under the Discovery Project Grants Scheme.

\section{References}

[1] R.M. German, Particle Packing Characteristics (Metal Powder Industries Federation Princeton New Jersey 1989.

[2] J.C. Maxwell, A Treatise on Electricity and Magnetism (Clarendon Press, Oxford 1892) (Vol. 1 pp 194-216).

[3] J.C. Maxwell-Garnett, Phil. Trans. Roy. Soc. Vol. 203 (1904), p. 386.

[4] J.R. Kalnin, E.A. Kotomin and J. Maier, J. Phys. Chem. Solids, Vol. 63 (2002), p. 449.

[5] J.R. Kalnin, E.A. Kotomin, J. Maier and V.N. Kuzovkov, in: Proceedings of Diffusion Fundamentals, edited by J. Kaerger, F. Grinberg and P. Heitjans, Leipzig, 2005, electronically published: www.uni-leipzig.de/diffusion/journal/pdf/volume2/diff_fund_2(2005)21.pdf

[6] I.V. Belova and G.E. Murch, J. Phys. Chem. Solids, Vol. 64 (2003), p. $9 \overline{73}$. 
Defects and Diffusion in Semiconductors - An Annual Retrospective IX doi:10.4028/3-908451-37-x

Monte Carlo Modelling of the Effective Diffusivity in Composite Material doi:10.4028/3-908451-37-x.103 